Article

\title{
Brewing Technique of Mbege, a Banana Beer Produced in Northeastern Tanzania
}

\author{
Ryosuke Kubo ${ }^{1, *}$ and Method Kilasara ${ }^{2}$ \\ 1 Graduate School of Global Environmental Studies, Kyoto University, Kyoto 606-8501, Japan \\ 2 Faculty of Agriculture, Sokoine University of Agriculture, Morogoro P.O. Box 3008, Tanzania; \\ mmkilasara@yahoo.com \\ * Correspondence: ryokubo1229@gmail.com; Tel.: +81-75-753-6101
}

Academic Editor: Shao Quan Liu

Received: 16 May 2016; Accepted: 26 July 2016; Published: 3 August 2016

\begin{abstract}
Mbege is a beer made of banana (Musa spp.) and finger millet (Eleusine coracana). It is the most popular indigenous alcoholic beverage in northeastern Tanzania, and plays an important role in the economy of the region. In this study, we observed and recorded a detailed traditional technique for brewing mbege. We observed that mbege production was divided into three major steps: nyalu preparation, mso preparation, and mixing. Fermented porridge made of ripened banana, called nyalu, was used as a source of yeasts. As a source of fermentable sugars, a sweet porridge made of germinated finger millet called $m s o$ was used. In $m s o$ preparation, a brewing technique to enhance the effectiveness of saccharification was used. After the preparation, these two types of porridge were mixed. The ethanol concentration of the mixture increased when it was fermented for $6 \mathrm{~h}$, and it then became mbege. It was supposed that yeasts in the nyalu converted fermentable sugars in the mso into ethanol. We found that the brewing technique used in the production of mbege in northeast Tanzania was similar to that used in southern Tanzania. We also demonstrated that the stem bark of Rauvolfia caffra, which was called msesewe and used as an additive in mbege production, accelerated the fermentation of nyalu and therefore increased the rate of ethanol production in the brewing of mbege. This result was consistent with the traditional knowledge in the field about the effect of msesewe on mbege production.
\end{abstract}

Keywords: Africa; alcoholic beverage; banana; brewing; food culture

\section{Introduction}

Indigenous alcoholic beverages are alcoholic beverages produced with locally available ingredients, instruments, and techniques. Although the production of indigenous alcoholic beverages has decreased globally in recent years due to the spread of mass-produced alcoholic beverages, indigenous alcoholic beverages are still popular in regions of Africa south of the Sahara desert. Statistically, about half of the total alcohol consumption in sub-Saharan Africa is in the form of indigenous alcoholic beverages [1]. In this region, these beverages take on socially, economically, and nutritionally important roles. They are served when people gather for social meetings in order to facilitate communication, and are also known to accompany religious and royal ceremonies [2-4]. In the case of cooperative work such as opening a field, weeding, and home building, it is served as an incentive and as a reward for work $[2,3,5,6]$. Producing indigenous alcoholic beverages is one of the precious few opportunities for women to earn a cash income [6,7]. They are also used as a source of nutrition and clean water [2,8]. Indigenous alcoholic beverages are necessary for people living in sub-Saharan Africa. Therefore, we must learn more about these beverages in order to further our understanding of the region. 
Tanzania is one of the sub-Saharan African countries in which indigenous alcoholic beverages are most abundantly produced and consumed. According to a report by the WHO, $87 \%$ of the total alcohol consumption in Tanzania comes in the form of indigenous alcoholic beverages [1]. This proportion of total consumption is more than 1.5 times larger than the average percentage of alcohol consumption attributed to indigenous alcoholic beverages in all countries of sub-Saharan Africa. More than 20 varieties of indigenous alcoholic beverages are found in Tanzania [9]. Among them, mbege, a banana beer made of banana (Musa spp.) and finger millet (Eleusine coracana), is the most popular indigenous alcoholic beverage in northeastern Tanzania [10]. However, in spite of its popularity and importance in the region, detailed brewing techniques of mbege had not yet been investigated prior to this study. Therefore, we carried out this study in order to clarify and record the brewing techniques of mbege.

\section{Materials and Methods}

\subsection{Field}

Field research activities such as recording observations, conducting interviews, taking measurements and performing on-site trials were carried out at Kilema kaskazini ward (9669 inhabitants divided into 2166 households) located in northeastern Tanzania [11]. The field research was carried out in July and November of 2015 and in January of 2016.

\subsection{Details of Mbege Production}

The details of the brewing technique of mbege were clarified by observation and interview. During the heating process in mbege production, changes in the temperature of the samples were measured using a handy infrared thermometer (826-T3; Testo, Kanagawa, Japan).

\subsection{Measurement of the Changes of Ethanol Concentration, Brix Value and $p H$ in Mbege Production}

The changes in ethanol concentration, Brix value and $\mathrm{pH}$ during mbege production were measured as described below. Ethanol concentration was measured by using a kit for measuring ethanol concentration in saliva (Q.E, $\mathrm{D}^{\circledR}$ Saliva Alcohol Test; OraSure Technologies Inc., Bethlehem, PA, USA). We diluted samples with water as needed before taking the measurement. We determined the Brix value of samples by using an Abbe refractometer (FHR-1; TGK, Tokyo, Japan). The $\mathrm{pH}$ values were measured using a handy $\mathrm{pH}$ meter (B-212; Horiba Ltd., Kyoto, Japan). All treatments and experiments were carried out on location using instruments we brought with us.

\subsection{On-Site Trial to Verify the Effect of Msesewe}

In northeastern Tanzania, stem bark of Rauvolfia caffra is used as an additive in mbege [12]. It is called msesewe in the Chagga language and used for mbege production (Figure 1). Msesewe was dried and ground into powder before being used for mbege production. People working in this region believed that it accelerated fermentation and increased the final ethanol concentration of mbege. The effect of msesewe on mbege production had not been verified prior to our study. Therefore, we investigated its effect by conducting an on-site trial.

In the trial, we prepared two types of mbege, one with and one without msesewe, each according to the brewing techniques we learned previously during the observation and interview phases of this study. Ethanol concentration, Brix value and $\mathrm{pH}$ of samples, which were measured during the brewing process, were obtained using the methods described above. These measured parameters of mbege batches brewed with and without msesewe were compared to verify the effect of msesewe on mbege production. 


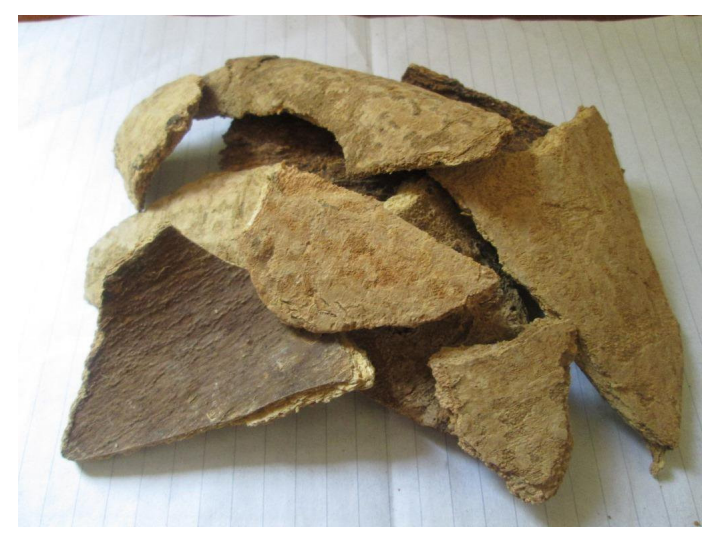

Figure 1. Msesewe.

\section{Results and Discussion}

\subsection{Varieties of Bananas Cultivated in the Research Field}

The banana is a fruit produced by several kinds of large herbaceous plants in the genus Musa. It is originally domesticated in southeastern Asia and brought into other parts of the world. In several parts of sub-Saharan Africa, it is eaten as a staple food. It is the most important crop as a staple food for the Chagga people in northeastern Tanzania. We found that there were 18 varieties of banana being cultivated within the research field (Table 1). Among them, ndishi was most frequently used for mbege production. According to the interview, the advantage of ndishi as an ingredient of mbege was that it was easily broken into small particles and became smooth when it was cooked. If banana was broken into small particles, the enzymes used for saccharification and the yeasts engaged in alcoholic fermentation could easily access the substrates of these reactions. Therefore, this characteristic of $n$ dishi might contribute to increasing the productivity of ethanol. Although ndishi was suitable for mbege production, it was not used for cooking purposes because of its unpleasant taste. In addition to ndishi, other types such as kimalindi, kipungara, kisukari, mchare, mrarao, and ndizi n'gombe were also used for mbege production (Table 1). Mchare, which was the most popular variety for cooking purposes in the field, was not used for mbege production frequently. The reason was that mchare required a lot of time and energy to cook if it was used for brewing, because it was not easily broken into small particles. However, people believed that the quality of mbege could be enhanced if mchare were used for its production, because it contained a larger amount of sugar than ndishi.

Table 1. Names and usage of banana varieties found in the research field.

\begin{tabular}{cc}
\hline Local Name & Usage \\
\hline Bokoboko & Cooking \\
Irongo & Cooking \\
Kimalindi & Fruit, cooking, brewing \\
Kinguruwe & Fruit, cooking, brewing \\
Kipungara & Brewing \\
Kisasa & Cooking \\
Kisukari & Fruit, brewing \\
Kitarasa & Cooking \\
Matoke ya Bukoba & Cooking \\
Matoke ya Mbeya & Cooking \\
Mchare & Cooking, brewing \\
Mkonosi & Cooking \\
Mnyenyele & Cooking \\
Mrarao & Fruit, brewing \\
Mzuzu & Cooking \\
Ndishi & Brewing \\
Ndizi n'gombe & Brewing \\
Ndizi Uganda & Cooking \\
\hline &
\end{tabular}




\subsection{Details of the Brewing Technique of Mbege}

The scheme of mbege production is shown in Figure 2. The production of mbege involved three major steps: nyalu preparation, mso preparation, and mixing. Details of these three steps are as follows.

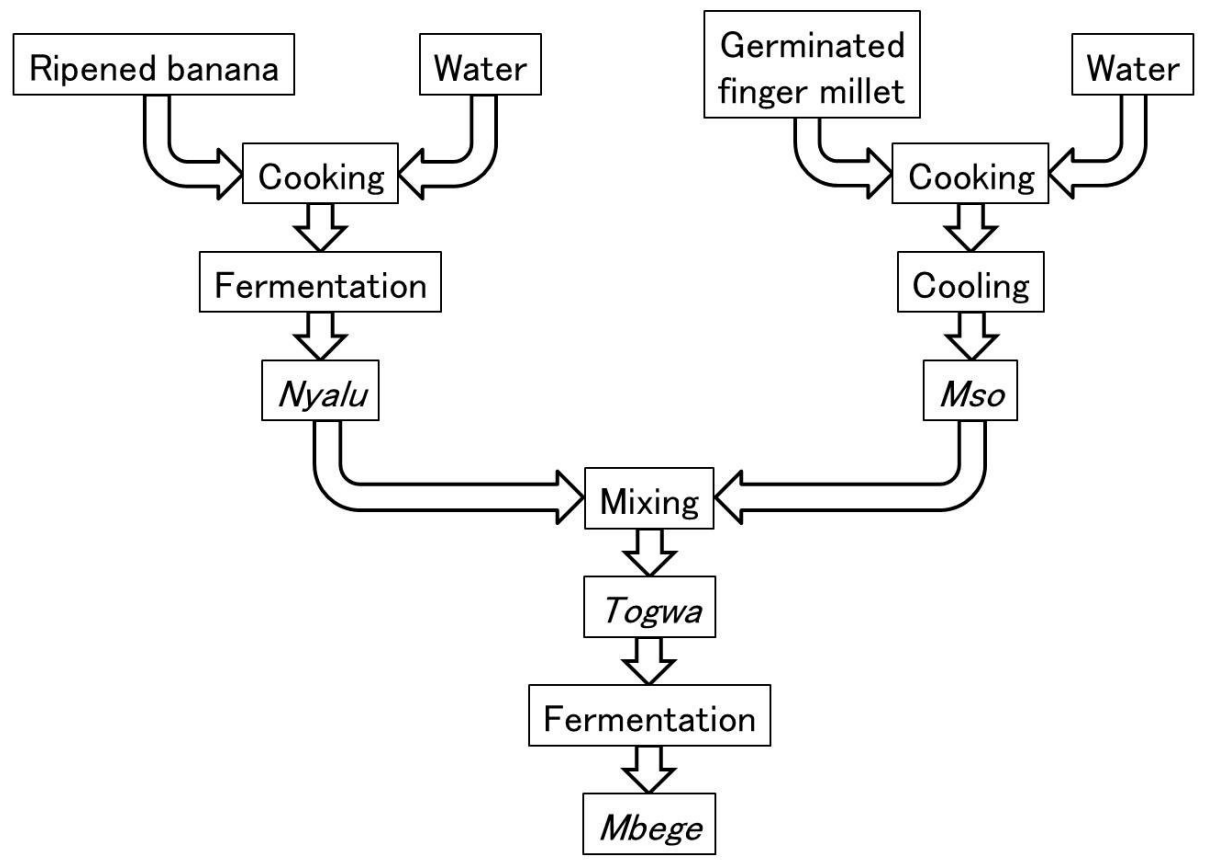

Figure 2. Scheme of mbege production.

Mbege production began with the preparation of nyalu. Nyalu was fermented porridge prepared with ripened banana. It was prepared as follows. First, $36 \mathrm{~L}$ of ripened banana was mixed with the same volume of water in an oil drum. The mixture was cooked over a high flame until its color became red and all the lumps were thoroughly broken down (Figure 3). After cooking, the mixture was transferred from the oil drum to a wooden barrel (Figure 4). It was then left to stand for 12 days at ambient temperature without covering. According to the brewer, the length of time required to ferment the cooked banana mixture varies with the season. Generally, the mixture is left to stand for nine to 10 days in the hot season and 12 to 13 days in the cold season. If the brewer uses msesewe for mbege production, a handful of it is added into the mixture after cooking. In this case, the mixture with msesewe will be ready after five days of fermentation. After a sufficient period of spontaneous fermentation, the mixture was filtered using a nylon bag (Figure 5). The mass leftover after the filtration step was called makati, and was utilized as feed for pigs. The obtained filtrate was the nyalu (Figure 6), a red porridge that had a slightly sour odor. According to our analysis, the ethanol concentration, Brix value and $\mathrm{pH}$ of nyalu were $1.2 \%, 5.6$ and 4.5, respectively. The presence of ethanol in nyaly indicated that yeasts were present in the mixture and spontaneous alcoholic fermentation had occurred. We observed bubbling at the surface of the cooked banana mixture during the fermentation stage. Its $\mathrm{pH}$ value suggested that nyalu could provide suitable conditions for yeasts to proliferate. By taking these facts into account, it was supposed that nyalu was the medium for yeast cultivation in mbege production. 


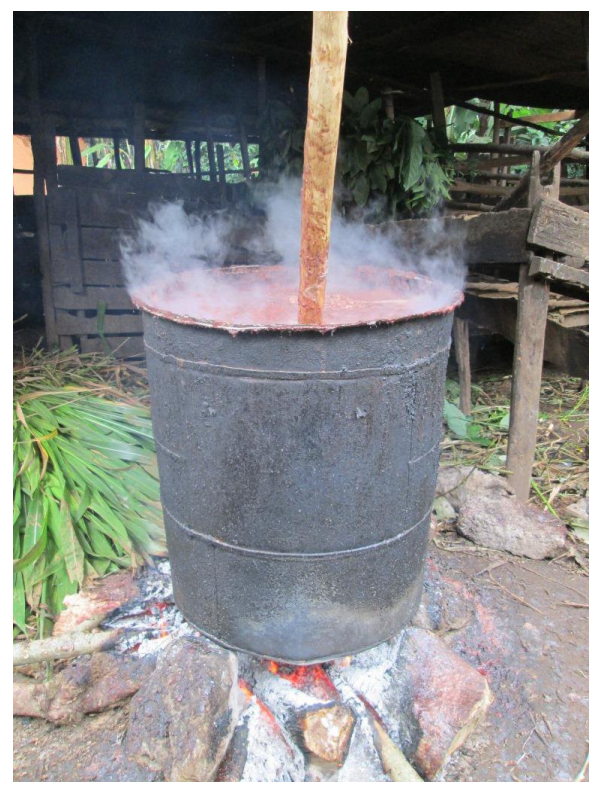

Figure 3. The mixture of ripened banana cooked on a high flame.

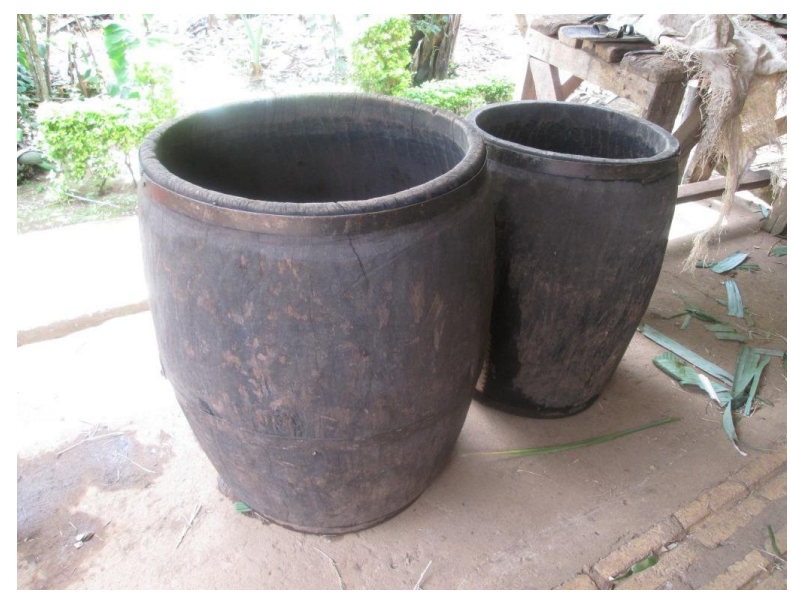

Figure 4. The wooden barrel to keep the cooked mixture.

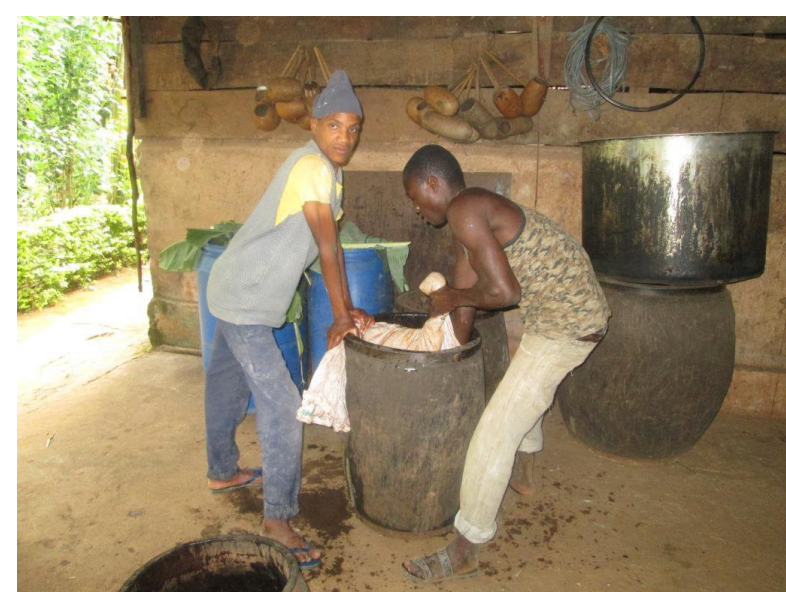

Figure 5. Filtering the fermented mixture using a nylon bag to obtain nyalu. 


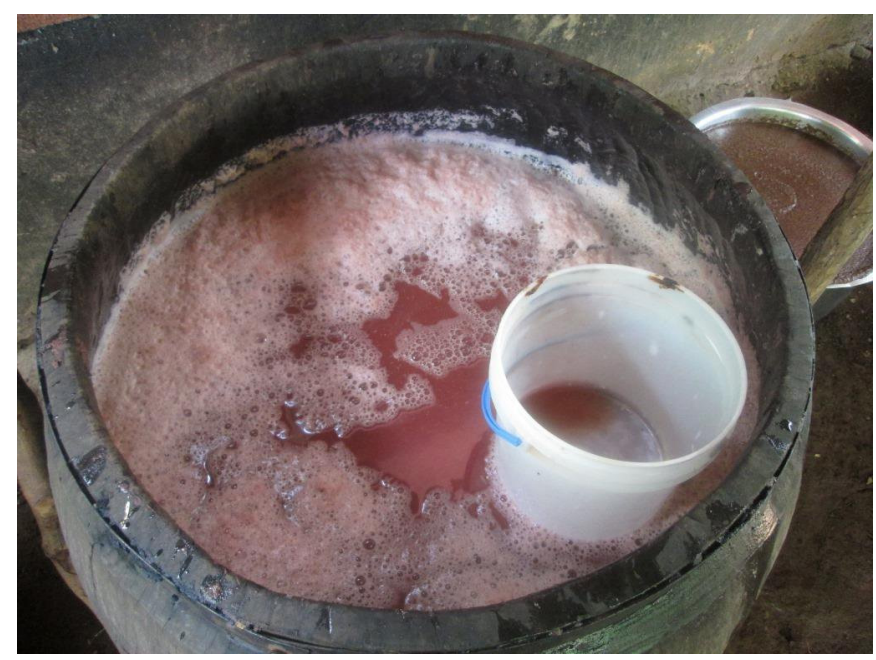

Figure 6. Nyalu.

After the nyalu was prepared, mso preparation began. Mso was porridge made of germinated finger millet. Mso was prepared according to the following process. First, $35 \mathrm{~L}$ of water was poured into a metal barrel, and then $5 \mathrm{~L}$ of germinated finger millet flour was added into the water. The barrel and its contents were heated over a fire for a few minutes, and once the temperature of the water had increased, $15 \mathrm{~L}$ of the warm, thin mixture was removed and put aside. This warm, thin mixture was used later to adjust the thickness of the main mixture. According to the brewer, the warm, thin mixture was used to thin out the main mixture instead of cold water so as to prevent a drop in temperature throughout the course of cooking. After removing the thin mixture, an additional $15 \mathrm{~L}$ of germinated finger millet flour was added into the main mixture cooking over the fire. The mixture was then stirred vigorously using a wooden rod with the occasional addition of the thin mixture (Figure 7). The mixture was cooked for $25 \mathrm{~min}$ with intermittent stirring after the incorporation of the second addition of germinated finger millet flour. The temperature of the mixture was checked carefully throughout the process, and was adjusted by adding or removing firewood. The brewer said that $m s o$ preparation could not be successful if the mixture was cooked at too-high temperatures. The change in the mixing temperature during cooking is shown in Figure 8. The result shows that the temperature was kept around $50-70{ }^{\circ} \mathrm{C}$. The amylase activity of finger millet is maximized at $50-60{ }^{\circ} \mathrm{C}$, but it is denatured beyond $70{ }^{\circ} \mathrm{C}$ [13]. Therefore, in mso preparation, the effectiveness of saccharification was maximized by keeping the temperature of the mixture in the range of $50-70{ }^{\circ} \mathrm{C}$. The same brewing technique was also observed in southern Tanzania [14]. Finally, the cooked mixture was transferred into several containers to let it cool. The obtained mixture was mso. Mso was a thick brown porridge with a sweet taste (Figure 9). The ethanol concentration, Brix value and $\mathrm{pH}$ of $m s o$ were $0 \%, 25.0 \%$ and $5.4 \%$, respectively. It did not contain ethanol. However, it showed a high Brix value. This meant that the mso was rich in fermentable sugars, which were generated by the action of amylase during cooking. The high amylase activity of germinated finger millet compared to other cereals cultivated in Tanzania might contribute to the high Brix value of $m s o$ [15]. It was suggested that mso was a source of fermentable sugars. 


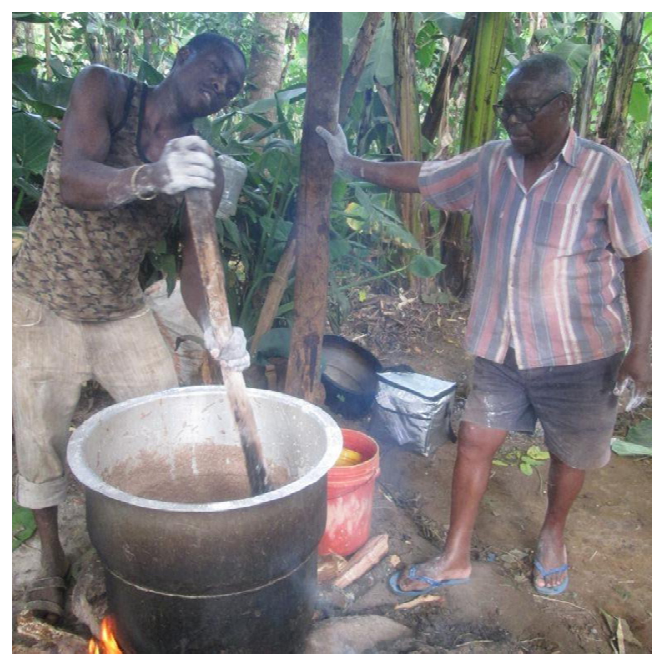

Figure 7. Stirring the mixture of germinated finger millet flour using a wooden rod.

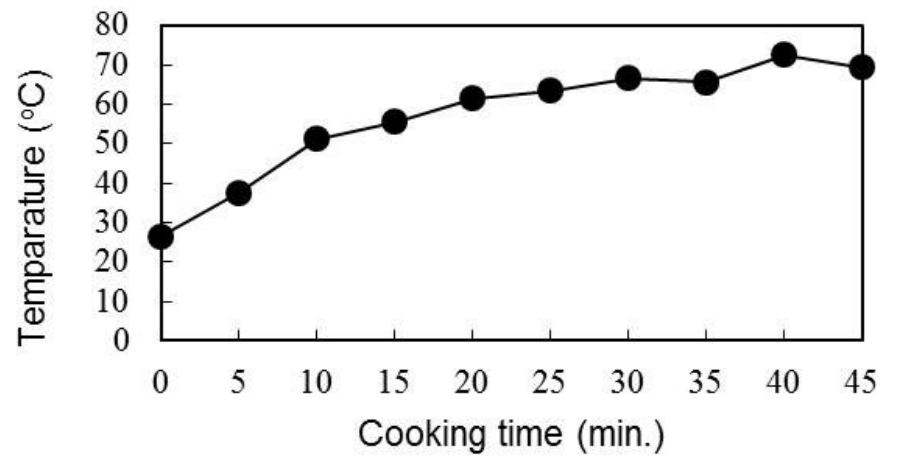

Figure 8. Change in the temperature of the germinated finger millet mixture during cooking.

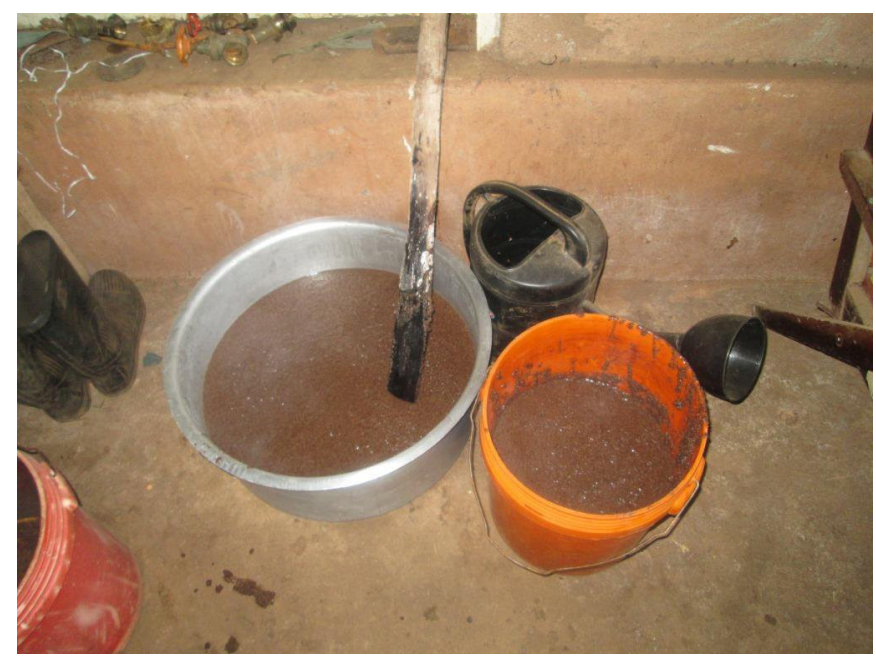

Figure 9. Mso.

Finally, the freshly prepared nyalu and mso were mixed together in a wooden barrel. As shown in Table 2, the mixture contained only a small amount of ethanol $(1.0 \%)$ right after the mixing process. The mixture was called togwa in this state. Togwa is the name of a saccharified beverage that is widely consumed in Tanzania [16]. It was a sweet and slightly sour beverage. After $6 \mathrm{~h}$ of 
fermentation, the ethanol concentration of the mixture increased to $2.7 \%$, and its Brix value decreased (Table 2). This was mainly the result of spontaneous alcoholic fermentation by yeasts in the nyalu using fermentable sugars from the mso. A yeast (Saccharomyces cerevisiae) and lactic acid bacterium (Lactobacillus plantarum) are the main microorganisms engaged in mbege production [10]. Therefore, lactic acid bacteria might also be engaged in the spontaneous fermentation in addition to yeasts, and might cause the decrease of the $\mathrm{pH}$ of the mixture. The mixture was called mbege in this state. Mbege was a red-colored alcoholic beverage with a slightly sour taste. A gourd cup was used to drink it (Figure 10). It was consumed within one or two days. The changes of the ethanol concentration, Brix value, and $\mathrm{pH}$ of the mbege over the course of $48 \mathrm{~h}$ of spontaneous fermentation after mixing are shown in Table 2. After $48 \mathrm{~h}$ of the fermentation, its ethanol concentration reached $4.0 \%$, and its Brix value dropped to 6.0. A similar brewing technique involving a mixture of two different types of porridge was also reported in southern Tanzania [14]. In that region, germinated finger millet is also used as an amylase source, and the brewing technique to control the temperature of the mixture during saccharification exists [14]. The results of this study showed that there were many brewing techniques shared between northeastern and southern Tanzania.

Table 2. Ethanol concentration, Brix value, and $\mathrm{pH}$ of togwa and mbege.

\begin{tabular}{cccc}
\hline & Ethanol Concentration (\%) & Brix & $\mathbf{p H}$ \\
\hline Togwa $(0 \mathrm{~h}) *$ & 1.0 & 9.0 & 4.6 \\
Mbege $(6 \mathrm{~h})$ & 2.7 & 8.0 & 4.4 \\
Mbege $(9 \mathrm{~h})$ & 3.0 & 8.5 & 4.4 \\
Mbege $(12 \mathrm{~h})$ & 3.2 & 7.5 & 4.2 \\
Mbege $(24 \mathrm{~h})$ & 3.0 & 6.5 & 4.2 \\
Mbege $(48 \mathrm{~h})$ & 4.0 & 6.0 & 4.2 \\
\hline
\end{tabular}

${ }^{*}$ Length of time (hours) after mixing nyalu and mso.

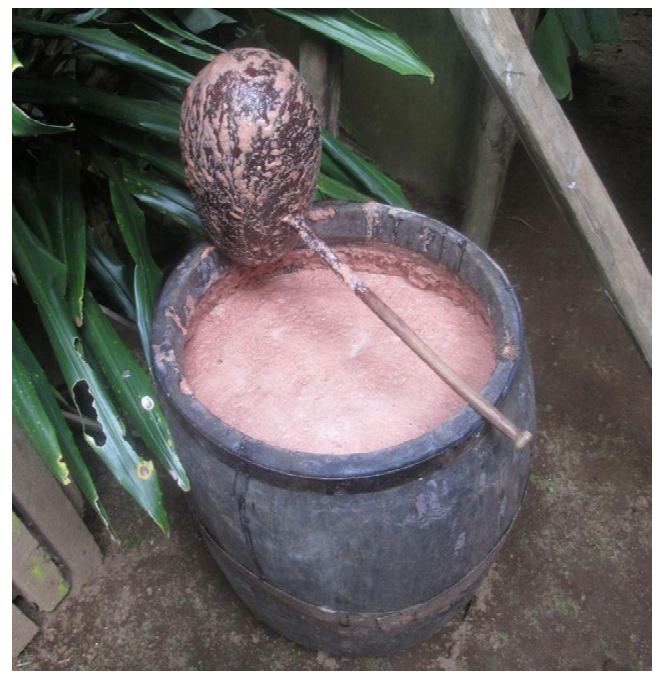

Figure 10. Mbege.

\subsection{The Effect of Msesewe on Mbege Production}

Under the direction of the local brewer, we prepared mbege with and without mesesewe, according to the brewing method shown in Figure 1. The ethanol concentration, Brix value and $\mathrm{pH}$ of the nyalu and mbege after $12 \mathrm{~h}$ of spontaneous fermentation were measured in each batch. The parameters of the samples are shown in Table 3. Our results showed that the ethanol concentration of the nyalu with msesewe was higher than that without msesewe. In contrast, the Brix value of the nyalu with msesewe was lower than that without msesewe. We also observed bubbling in the nyalu with msesewe 
after five days of spontaneous fermentation, although it was not observed in that without msesewe. These facts suggested that the alcoholic fermentation had taken place much more actively in the nyalu with msesewe than in that without msesewe. It was reported that the stem bark of mangroves can suppress the growth of all microorganisms in palm wine except for yeasts [17]. This suppressive effect is due to the tannins in the bark. As msesewe also contains tannins, it was supposed that msesewe helped yeasts to become the dominant microorganism in nyalu [12]. Yeasts might be able to proliferate rapidly once they dominate the population of microorganisms in nyalu. Our observations were consistent with the traditional understanding that msesewe accelerated the fermentation process.

Table 3. Ethanol concentration, Brix value, and $\mathrm{pH}$ of nyalu and mbege with msesewe (A) and that without msesewe (B).

\begin{tabular}{|c|c|c|c|}
\hline & Ethanol Concentraion (\%) & Brix & $\mathrm{pH}$ \\
\hline \multicolumn{4}{|c|}{ A. Mbege with mesesewe } \\
\hline Nyalu & 0.7 & 10.0 & 4.7 \\
\hline Mbege $(12 \mathrm{~h})$ * & 4.2 & 9.0 & 4.7 \\
\hline \multicolumn{4}{|c|}{ B. Mbege without mesesewe } \\
\hline Nyalu & 0.2 & 15.0 & 4.6 \\
\hline Mbege $(12 \mathrm{~h})$ * & 1.8 & 11.0 & 4.6 \\
\hline
\end{tabular}

Similarly to the case of nyalu, the ethanol concentration of mbege with msesewe after $12 \mathrm{~h}$ of spontaneous fermentation was higher than that without msesewe, and their Brix values showed the opposite relationship (Table 3). This result was also consistent with the traditional knowledge of brewers in this region, which stated that msesewe increased the ethanol concentration of mbege. It was supposed that the ethanol concentration of mbege with msesewe became higher than that without msesewe, because nyalu with msesewe contained a much larger population of yeasts capable of spontaneous alcoholic fermentation. In this on-site trial, we demonstrated the validity of the traditional understanding of the effect of msesewe on mbege production. However, an excess amount of msesewe may cause inefficient alcoholic fermentation and an unpalatable taste of mbege because of the effect of tannins [18]. Therefore, we must determine the adequate range of the quantity of msesewe to add for improving the quality of mbege in a future study.

\section{Conclusions}

This study observed and recorded a detailed traditional brewing technique of mbege. Mbege was produced by mixing two different porridges, nyalu and $m s o$. The nyalu was a fermented porridge made of ripened banana. It was the source of yeasts in mbege production. The other ingredient, mso, was a sweet porridge made of germinated finger millet. Its role was as a source of fermentable sugars. In the preparation of $m s o$, we observed the technique to maintain the temperature of the mixture within a specific range for optimized saccharification. We found that the brewing techniques of mbege in northeastern Tanzania had similarities with the techniques of southern Tanzania.

We also verified the effect of msesewe on mbege production. The on-site trial found that msesewe accelerated the fermentation of nyalu and increased the final ethanol concentration of mbege. These effects of msesewe were consistent with the traditional knowledge of this region. Therefore, we demonstrated the validity of this traditional knowledge in regards to the effect of msesewe through the on-site trial. 
Acknowledgments: We would like to thank the people in the Kilema kaskazini ward for their all kindness during our stay. We would also like to extend our appreciation to all the members of our research project, the International Network-hub for Future Earth: Research for Global Sustainability, for their encouragement to complete this study. This study was granted by the Program for Advancing Strategic International Networks to Accelerate the Circulation of Talented Researchers and promoted by the Japan Society for the Promotion of Science (JSPS).

Author Contributions: In this study, Ryosuke Kubo conceived and designed the field research and experiments; Ryosuke Kubo and Method Kilasara conducted the field research together; Ryosuke Kubo performed the experiments in the field; Ryosuke Kubo wrote the paper under the supervision of Method Kilasara.

Conflicts of Interest: The authors declare no conflict of interest.

\section{References}

1. World Health Organization (WHO). Global Status Report on Alcohol and Health 2014. Available online: http://www.who.int/substance_abuse/publications/global_alcohol_report/msb_gsr_2014_2.pdf? ua $=1$ (accessed on 10 November 2014).

2. Madovi, P.B. Food handling in Shona village of Zimbabwe. Ecol. Food Nutr. 1981, 11, 133-144. [CrossRef]

3. Benhura, M.A.; Chingombe, A. Traditional brewing methods in Zimbabwe. Zimbabwe Sci. News 1989, 23, 69-70.

4. Willis, J. Potent Brews: A Social History of Alcohol in East Africa 1850-1999; Ohio University Press: Athens, OH, USA, 2002; p. 304.

5. Platt, B.S. Some traditional alcoholic beverages and their importance in indigenous African communities. Proc. Nutr. Soc. 1955, 14, 115-124. [CrossRef] [PubMed]

6. Kakeya, M.; Sugiyama, Y. Citemene, Finger Millet and Bemba Culture: A Socio-ecological Study of Slash-and-burn Cultivation in Northeastern Zambia. Afr. Stud. Monogr. 1985, 4, 1-24.

7. McCall, M. Brewing rural beer should be a hotter issue. Boil. Point 2001, 47, 23-25.

8. Sunano, Y. Use of the alcoholic beverage Parshot as staple food in Dirache area, southern Ethiopia with emphasis on the nutritive value and intake of alcoholic beverages. Res. Trop. Agric. 2013, 6, 69-74. (In Japanese)

9. Tiisekwa, B. Traditional beers processing in Tanzania: Development needs. Afr. Focus 1986, 2, $299-324$.

10. Shayo, N.B.; Nnko, S.A.M.; Gidamis, A.B. Isolation and characterisation of yeasts and bacteria from mbege-An opaque beer made from millet malt and banana juice. Tanzania J. Agric. Sci. 1998, 1, 57-63.

11. Tanzania National Bureau of Statistics (NBS). United Republic of Tanzania Census 2012. Available online: http://50.87.153.5/ eastc/sensa/index.php/home/BookOneDo (accessed on 9 February 2016).

12. Njau, E.A.; Alcorn, J.; Ndakidemi, P.; Chirino-Trejo, M.; Buza, J. Antimicrobial and antioxidant activity of crude extracts of Rauvolfia caggre var. caffra (Apocynaceae) from Tanzania. Int. J. Biol. 2014, 6, 156-167. [CrossRef]

13. Gimbi, D.M.; Kitabatake, N. Changes in alpha-and beta-amylase activities during seed germination of African finger millet. Int. J. Food Sci. Nutr. 2002, 53, 481-488. [CrossRef] [PubMed]

14. Kubo, R. Production of indigenous alcoholic beverages in a rural village of Tanzania. J. Inst. Brew. 2014, 120, 142-148. [CrossRef]

15. Kubo, R. The reason for the preferential use of finger millet (Eleusine coracana) in eastern African brewing. J. Inst. Brew. 2016, 122, 175-180. [CrossRef]

16. Kitabatake, N.; Gimbi, D.M.; Oi, Y. Traditional non-alcoholic beverage, Togwa, in East Africa, produced from maize flour and germinated finger millet. Int. J. Food Sci. Nutr. 2003, 54, 447-455. [CrossRef] [PubMed]

17. Nishiyama, R.; Sanchez, P.C.; Kozaki, M. Inhibitory function of mangrove bark toward cell growth of microorganisms. Hakkokogaku 1978, 56, 712-717. (In Japanese)

18. Dykes, L.; Rooney, L.W. Phenolic compounds in cereal grains and their health benefits. Cereal Food World 2007, 52, 105-111. [CrossRef]

(C) 2016 by the authors; licensee MDPI, Basel, Switzerland. This article is an open access article distributed under the terms and conditions of the Creative Commons Attribution (CC-BY) license (http://creativecommons.org/licenses/by/4.0/). 